\title{
恒河猕猴吗啡成瘾模型的初步建立
}

\author{
吴绪军 ${ }^{1,2}$, 宗 慰 ${ }^{1,2}$, 孙永梅 ${ }^{1,2}$, 胡新天 ${ }^{1}$, 马原野 ${ }^{1}$, 王建红 ${ }^{1, *}$ \\ (1. 中国科学院昆明动物研究所, 云南 昆明 650223; 2. 中国科学院研究生院, 北京 100049)
}

\begin{abstract}
摘要: 吗啡成瘾的非人灵长类动物恒河狝猴(Macaca mulatta)模型的实验结果表明, 猕猴可建立吗啡条件化位 置偏好 (conditioned place preference, CPP), 且其与吗啡线索相关的记忆可持续(36.3 \pm 1.3 )月。该研究可以为药物成 㒣研究提供有效的非人灵长类动物行为模型。
\end{abstract}

关键词: 吗啡; 猕猴; 条件化位置偏好

中图分类号: Q959.832; Q959.848; R-331

文献标志码：A＼cjkstart文章编号：0254-5853-(2012)01-0089-03

\section{Morphine addiction in non-human primates using rhesus monkeys}

\author{
WU Xu-Jun ${ }^{1,2}$, ZONG Wei ${ }^{1,2}$, SUN Yong-Mei ${ }^{1,2}$,HU Xin-Tian ${ }^{1}$, MA Yuan-Ye ${ }^{1}$, WANG Jian-Hong ${ }^{1, *}$ \\ (1.Kunming Institute of Zoology, the Chinese Academy of Sciences, Kunming 650223, China; \\ 2.Graduate School of the Chinese Academy of Sciences, Beijing 100049, China)
}

\begin{abstract}
We showed that rhesus monkeys (Macaca mulatta) can develop a morphine dependence. Rhesus monkeys successfully established a conditioned place preference (CPP) induced by morphine treatment and this preference lasted for at least $(36.3 \pm 1.3)$ months. This animal model may be useful for research into addiction in humans.
\end{abstract}

Key words: Morphine; Macaca mulatta; Conditioned place preference

药物成滤是当今社会的一大危害，对成瘾药物 戒断的研究已成为当务之急。以往药物成瘾研究多 采用啮齿类动物模型。一旦持续性给予动物吗啡、 可卡因等成滤药物，动物将对药物产生依赖性；而 一旦停药, 动物将出现戒断症状, 从而迫使动物寻 求药物。利用这一模式, 可将动物对药物的寻求过 程与相关环境线索联系起来, 以检测动物对成瘾药 物的渴求程度。

以啮齿类动物为研究对象, 常采用条件化位置偏 好(conditioned place preference, CPP)检测法, 该方 法利用动物对与药物相关信息的记忆, 可有效反映 动物对药物的成瘾及渴求状态(Hnasko et al, 2005; Rice et al, 2002; Vindenes et al, 2006; Marquez et al, 2009)。

利用小鼠 CPP 模式, 我们发现, 阻断乙酰胆碱 系统, 或阻断大脑蛋白质的合成, 以及电休克、戊
四氮致癫㾁等干预，都不能损伤与成瘾相关的学习 记忆(Tan et al, 2007; Zhang et al, 2008)。结合其他实 验室的研究结果, 我们认为药物成瘾是一种异常、 病态的学习过程, 建立有效的药物成㒣动物模型, 将有助于为人类药物成瘫的戒断寻找新的策略。

药物成滤研究常采用啮齿类动物模型, 有关非 人灵长类动物模型的数据非常有限。非人灵长类动 物的神经生物学研究是本实验室及中国科学院和 云南省动物模型与人类疾病机理重点实验室的优 势, 因而, 我们以恒河猕猴(Macaca mulatta) 为研究 对象,采用 $\mathrm{CPP}$ 模式, 探讨这种非人灵长类动物对 吗啡成瘾、戒断及相关线索的学习记忆, 以期为研 究药物成瘾提供新的动物模型。

\section{1 恒河猕猴吗啡成瘾及 CPP 相关学习记忆}

Foltin \& Evans(2001)采用两箱装置, 发现猕猴

收稿日期: 2011-12-19; 接受日期: 2012-01-05

基金项目：973 项目(2011CB707800)和中国科学院知识创新工程重要方向项目(KSCX2-EW-J-23)

*通信作者(Corresponding author), E-mail: wangjh@mail.kiz.ac.cn 
可以对海洛因烟雾产生位置偏好。在这一模型中, 猕猴只能在两个箱子之间选择，缺乏提供狝猴思考 和抉择的中性过渡箱。因而, 我们建立了适用于猕 猴的三箱 CPP 模型。该标准三箱设备具封闭通道, 各通道具可开、关的门。狝猴在三箱设备内的活动 通过摄像头进行监控和录像。采用视频采集软件记 录及人工分析的方法，可评价动物穿梭三箱的次 数、时间, 及在各个箱内的活动情况(图 1)。

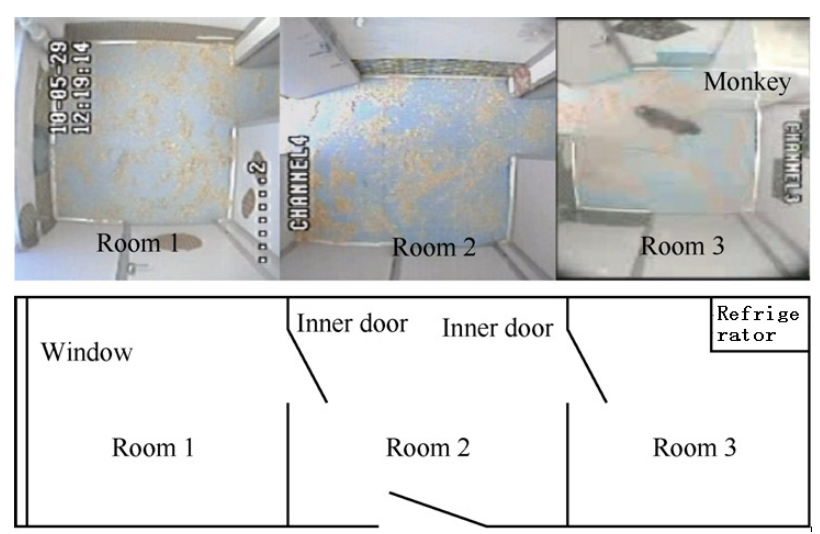

图 1 猕猴 CPP 模型

Fig.1 Monkey model of CPP

左侧(箱 1)和右侧(箱 3)尺寸: $190 \mathrm{~cm} \times 190 \mathrm{~cm} \times 265 \mathrm{~cm}$; 中间过渡箱(箱 2)尺寸： $210 \mathrm{~cm} \times 210 \mathrm{~cm} \times 265 \mathrm{~cm}$ (长×宽 $\times$ 高)。左侧箱内有一扇贴膜 的窗子, 右侧箱内有一电冰箱 (Refrigerator), 分别作为箱体的标记(Wang et al, 2011)。

The left (room 1) and right (room3) were $190 \mathrm{~cm} \times 190 \mathrm{~cm} \times 265 \mathrm{~cm}$ (length $\times$ width $\times$ height); the intermediate room(room2)size was $210 \mathrm{~cm} \times$ $210 \mathrm{~cm} \times 265 \mathrm{~cm}$. There was a covered window in the room 1 and a refrigerator in the room3 as different visual cues (Wang et al, 2011).

\section{1 实验步骤}

适应：条件化之前 $1 \sim 3 \mathrm{~d}$ ，使狝猴充分适应 3 个箱体 $50 \mathrm{~min}$, 记录并统计数据。将狝猴不偏爱的 箱体, 设为与吗啡相关的阳性箱, 即注射吗啡后猕 猴停留的箱体; 而将猕猴自然偏爱箱设为药物阴性 箱，即注射生理盐水后猕猴停留的箱体。

CPP 条件化建立(共 $12 \mathrm{~d}$ ): 给狝猴肌肉注射吗 啡(递增式剂量: $1.5 \mathrm{mg} / \mathrm{kg}$ 体重 $\times 1 \mathrm{~d} 、 3.0 \mathrm{mg} / \mathrm{kg}$ 体 重 $\times 1 \mathrm{~d} 、 4.5 \mathrm{mg} / \mathrm{kg}$ 体重 $\times 4 \mathrm{~d})$, 每次注射完毕，使

\section{参考文献:}

Foltin RW, Evans SM. 2001. Location preference related to smoked heroin self-administration by rhesus monkeys[J]. Psychopharmacology: Berl, 155: 419-425.

Hnasko TS, Sotak BN, Palmiter RD. 2005. Morphine reward in dopamine-deficient mice[J]. Nature, 438: 854-857.

Marquez P, Bebawy D, Lelievre V, Coute AC, Evans CJ, Waschek JA, Lutfy K. 2009. The role of endogenous PACAP in motor stimulation and
猕猴在阳性箱内停留 $50 \mathrm{~min}$, 促其建立与吗啡相关 的记忆; 每次吗啡注射 $24 \mathrm{~h}$ 后，给猕猴注射相同体 积生理盐水，之后使猕猴在阴性箱内停留 $50 \mathrm{~min}$, 使其建立与生理盐水相关的记忆。

CPP 条件化检测: 条件化之后 $24 \mathrm{~h} 、(15.8 \pm 0.8)$ 月和(36.3 \pm 1.3$)$ 月, 进行 CPP 检测, 各连续 $2 \mathrm{~d}$ 。此 时, 3 个箱体之间的门均敞开, 猕猴可自由选择停留 的箱子。记录猕猴第一次选择的箱体，及 $0 \sim 5 、 5 \sim$ 20 和 20 30 min 内猕猴在三个箱子里的停留时间 和访问次数, 以及行走距离。

\section{2 实验结果}

6 只猕猴均在短期内建立吗啡 CPP, 且无药效以 外的不良反应。此外, 在间隔 15.8 (6 只狝猴)和 36.3 (3 只猕猴) 个月之后, 猕猴仍保存对吗啡相关线 索的记忆, 表现为在阳性箱内停留时间、访问次数 和行走距离均明显高于阴性箱内的对应参数。说明 吗啡相关线索的记忆能够在猕猴上快速建立并长 期维持。

有趣的是，随着检测时间间隔的延长，猕猴在 中间过渡箱内停留的时间增长，推测猕猴在条件化 $24 \mathrm{~h}$ 之后, 对吗啡的渴求很强, 因而进入阳性箱的 时间和次数增高。然而，一段时间间隔之后再检测， 猕猴因为无法在阳性箱内得到吗啡, 为了避免渴求 而不能得到药物的厌恶情绪，故选择在中间的中性 箱停留。但是，与其他两个箱体相比，猕猴仍然偏 爱停留在阳性箱。

该实验结果说明, 在 6 次递增剂量吗啡注射之 后，猕猴对与吗啡相关线索的记忆持续时间至少可 达(36.3 \pm 1.3$)$ 月。该模型具备良好的操作性和稳定 性, 可为研究药物成瘾的机制和戒断策略提供可靠 且更接近人类的动物模型。

致谢: 感谢中国科学院昆明动物研究所研究生 李传玉、魏景宽、姜慧慧、刘赐融、于程洋、马骅 等对实验的贡献。

conditioned place preference induced by morphine in mice[J]. Psychopharmacology: Berl, 204: 457-463.

Rice OV, Gordon N, Gifford AN. 2002. Conditioned place preference to morphine in cannabinoid CB1 receptor knockout mice[J]. Brain Res, 945: $135-138$.

Tan H, Liu N, Wilson FA, Ma Y. 2007. Effects of scopolamine on morphine-induced conditioned place preference in mice[J]. Addict Biol, 
12: 463-469.

Vindenes V, Handal M, Ripel A, Boix F, Morland J. 2006. Conditioned place preference induced by morphine and morphine-6-glucuronide in mice[J]. Pharmacol Biochem Behav, 85: 292-297.

Wang JH, Wu XJ, Li CY, Wei JK, Jiang HH, Liu CR, Yu CY, Carlson S, Hu XT, Duan W, Ma YY. 2011. Effect of morphine on conditioned place preference in rhesus monkeys[J]. Addict

Biol, doi: 0.1111/j.1369-1600.2010.00289.x. (Online version).

Zhang J, Tan H, Niu HC, Wang JH, Tang XD, Sanford LD, Ma YY. 2008. Pentylenetetrazole-induced status epilepticus following training does not impair expression of morphine-induced conditioned place preference[J]. Addict Biol, 14: 174-184.

\section{中国科学院/云南省动物模型与人类疾病机理重点实验室最新进展}

中国科学院/云南省动物模型与人类疾病机理重点实验室（以下简称实验室）的前身是中国科学院昆 明动物研究所所级重点实验室，成立于2005年12月20日。2011年，该实验室在学术委员会的指导下，全体 人员抢抓机遇、开拓进取, 积极发挥资源优势和研究特色, 在科学研究、平台建设、经费申请、队伍建设 与人才培养等方面均取得了较大进展, 实现了“十二五”开门红。

科研方面, 立足当前、谋划长远, 实现了基础研究与应用研究并举, 科研发展呈现出强劲的势头。一 年来, 实验室整体科研产出在 2010 年的基础上更进一步, 取得了一批具有国际影响力的研究成果, 特别是 完成了树鼣全基因组测序，为动物模型创制和重大疾病机理研究奠定了坚实的基础。

实验室主要围绕疾病动物模型与实验动物创制、疾病机理解析和新药研发、药用动物天然活性物质的 结构与功能开展工作。2011年共发表相关论文97篇, 其中第一单位论文55篇, 第一单位SCI论文37篇, IF >5 的论文16篇; 参编专著2部。2011年实验室共申请专利13项, 获得授权专利4项; 抗抑郁1类新药-奥生乐赛 特获得国家食品药品监督管理局（SFDA）颁发的药物I、II、III期临床试验批件。此外，2011年度实验室 在动物模型研究及平台建设方面也取得了重大进展。

2011年实验室成功申请国家、省部级、国际合作等项目 35项，其中主持27项，参加8项，新增科研经 费3200余万元。

实验室凝聚和建设了一支能够在多学科交叉和国际前沿进行拼搏、又能为地方特色生物资源开发利用 和产业化提供科技支撑的科研团队。2011年实验室共培养毕业研究生22人，其中博士11人，硕士11人; 接 收客座研究人员 54 人；2人入选 “云南省中青年学术与技术学科带头人后备人才”; “肿瘤分子标记与抗 肿瘤药物研究”团队成功入选云南省创新团队，极大地提升了实验室的科研实力和集团竞争力。

展望2012年，实验室将在主管部门与依托单位的关心支持下，在学术委员会的指导下，面向国家重大 战略需求, 立足于研究所“一三五”发展规划, 依托 “中国科学院昆明灵长类动物中心”、“中国科学院 树鼣饲养繁殖基地”、“昆明国家生物产业基地灵长类实验动物中心”、“国家昆明高等级生物安全灵长 类动物实验中心”，发扬优势，集群创新，联合攻关，争取建成有国际水准、面向国内外的非人灵长类动 物模型研究基地和公共服务平台, 为提升我国重大疾病研究、新药研发等方面的自主创新能力和国际竞争 能力，满足我国人口与健康领域的迫切、重大需求做出贡献!

中国科学院/云南省动物模型与人类疾病机理重点实验室 (中国科学院昆明动物研究所, 云南 昆明 650223) 